hep-ph/9810236

NT@UW-98-23

1st October 1998

\title{
The Soft Régime and $\beta$ Function of NRQCD
}

\author{
Harald W. Grießhammer円 \\ Nuclear Theory Group, Department of Physics, University of Washington, \\ Box 351 560, Seattle, WA 98195-1560, USA
}

\begin{abstract}
Progress towards a complete velocity power counting in non-relativistic effective field theories, especially NRQCD, is motivated and summarised.
\end{abstract}

\footnotetext{
${ }^{1}$ Talk presented at the "Euroconference QCD '98" in Montpellier, France, 2nd - 8th July 1998 (to appear in Nucl. Phys. B(Proc. Suppl.)), and at the conference "Quark Confinement and the Hadron Spectrum III" at TJNL, Newport News, USA, 8th - 12th June 1998 (to appear in the proceedings). Email: hgrie@phys.washington.edu
} 
In Non-Relativistic QCD [1, 2], a heavy quark is nearly on shell and its energy is dominated by its mass $M$, so that the resulting non-relativistic system can be treated perturbatively both in the coupling constant $g$ and in the quark velocity $v$. The typical energy and momentum scales are the non-relativistic kinetic energy $M v^{2}$ and the momentum $M v$. The effective Lagrangean does not exhibit $v$ explicitly, so that a power counting scheme has to be established which determines uniquely which terms in the Lagrangean must be taken into account to render predictive power to a given precision in $v$.

Identification of the relevant energy and momentum régimes has proven difficult: The first attempt by Lepage and co-workers [3] fell shot as working only in the Coulomb gauge and as being incomplete. In a recent article, Beneke and Smirnov [4 pointed out that the much simpler velocity rescaling rules proposed by Luke and Manohar for Coulomb interactions [5], and by Grinstein and Rothstein for bremsstrahlung processes [6], as united by Luke and Savage [7], and by Labelle's power counting scheme in time ordered perturbation theory [8], do not reproduce the two gluon exchange between particles near threshold. Recently, velocity power counting rules using dimensional regularisation were established for a toy model [9] and NRQCD [10] (see also [11]) following Beneke and Smirnov's threshold expansion [⿶]. Here, I summarise two recent publications [9, 10].

The NRQCD propagators are read off from the Lagrangean as

$$
Q: \frac{\mathrm{i} \mathrm{Num}}{T-\frac{\vec{p}^{2}}{2 M}+\mathrm{i} \epsilon}, A^{\mu}: \frac{\mathrm{i} \mathrm{Num}}{k^{2}+\mathrm{i} \epsilon},
$$

where $T=\frac{\vec{p}^{2}}{2 M}+\ldots$ is the kinetic energy of the quark. "Num" are numerators containing the appropriate colour, Dirac and flavour indices. Cuts and poles in scattering amplitudes stem from bound states and on-shell propagation of particles in intermediate states and give rise to infrared divergences. With energies and momenta of either scale, there are only three régimes in which quarks or gluons in (1) are on shell:

$$
\begin{array}{rll}
\text { soft régime: } & A_{\mathrm{s}}^{\mu}: & k_{0} \sim|\vec{k}| \sim M v, \\
\text { potential régime: } & Q_{\mathrm{p}}: & T \sim M v^{2},|\vec{p}| \sim M v \\
\text { ultrasoft régime: } & A_{\mathrm{u}}^{\mu}: & k_{0} \sim|\vec{k}| \sim M v^{2}
\end{array}
$$

Ultrasoft gluons $A_{\mathrm{u}}^{\mu}$ are emitted as bremsstrahlung or from excited states in the bound system, and hence physical. Overall energy conservation forbids all processes with outgoing soft gluons but without ingoing ones. Gluons which change the quark momenta but keep them close to their mass shell relate the (instantaneous) Coulomb interaction:

$$
A_{\mathrm{p}}^{\mu} \quad: \quad k_{0} \sim M v^{2},|\vec{k}| \sim M v
$$

When a soft gluon $A_{\mathrm{s}}^{\mu}$ couples to a potential quark $Q_{\mathrm{p}}$, the outgoing quark is far off its mass shell and carries energy and momentum of order $M v$.

$$
Q_{\mathrm{s}}: T \sim|\vec{p}| \sim M v
$$


As the potential quark has a much smaller energy than either of the soft particles, it can - by the uncertainty relation - not resolve the precise time at which the soft quark emits the soft gluon. So, a "temporal" multipole expansion is associated with this vertex. In general, the coupling between particles of different régimes will not be point-like but contain multipole expansions for the particle belonging to the weaker kinematic régime.

One may expand [4] the integrand of a loop integral in NRQCD about the various saddle points, i.e. about the values of the loop-momentum $q$ where particles become on shell. For example, expanding about a physical gluon at the soft scale, the quark propagator is $\left(T_{\mathrm{p}} \sim \frac{\vec{p}^{2}}{2 M} \sim M v^{2} \ll q_{0} \sim|\vec{q}| \sim M v\right)$

$$
\frac{\mathrm{i}}{q_{0, \mathrm{~s}}+T_{\mathrm{p}}-\frac{(\vec{p}+\vec{q})^{2}}{2 M}} \longrightarrow \frac{\mathrm{i}}{q_{0, \mathrm{~s}}}+\frac{\mathrm{i}}{q_{0, \mathrm{~s}}} \mathrm{i}\left(T_{\mathrm{p}}-\frac{(\vec{p}+\vec{q})^{2}}{2 M}\right) \frac{\mathrm{i}}{q_{0, \mathrm{~s}}}+\ldots .
$$

Higher order terms in the expansion are interpreted as insertions into the soft quark propagator (static to lowest order) and as multipole expansions which modify the vertex rules. As the energy of potential gluons is much smaller than their momentum, the $A_{\mathrm{p}^{-}}$ propagator becomes instantaneous. With these five fields $Q_{\mathrm{s}}, Q_{\mathrm{p}}, A_{\mathrm{s}}^{\mu}, A_{\mathrm{p}}^{\mu}, A_{\mathrm{u}}^{\mu}$ representing the relevant infrared-relevant degrees of freedom, i.e. quarks and gluons in the three different non-relativistic régimes, soft, potential and ultrasoft, NRQCD becomes selfconsistent.

The quark propagator is gauge independent. Gauge fixing introduces gauge dependent denominators multiplying the gauge independent denominators in the gluon propagators. When they are non-zero for all but the above combinations of scales $M v$ and $M v^{2}$ (2), the decomposition into the three régimes (2) remains unchanged, as do the rescaling properties of the fields and interactions. The standard gauges (axial, Weyl, Lorentz, Coulomb) will therefore all show the same power counting and vertex rules. Details of the gluon propagator and its insertions are different in different régimes and gauges, and some gauges will not exhibit certain vertices, insertions and representatives; e.g. the Coulomb gauge is unique in having $A_{0}$ contribute only in the potential régime as physical fields are transverse by virtue of Gauß' law.

Finally, the regularisation scheme must be chosen such that expansion around one saddle point in the loop integral does not obtain any contribution from other saddle points. Cutoff regularisation jeopardises power counting and symmetries, and introduces unphysical power divergences. In contradistinction, using dimensional regularisation after the saddle point expansion preserves power counting and gauge symmetry. Its homogeneity guarantees that contributions from different saddle points and régimes do not overlap. (A simple example is given in Ref. [9].)

In order to establish explicit $v$ counting in the NRQCD Lagrangean, one rescales the space-time coordinates such that typical momenta in each régime are dimensionless:

$$
\begin{aligned}
\text { soft: } & t=(M v)^{-1} T_{\mathrm{s}}, \vec{x}=(M v)^{-1} \vec{X}_{\mathrm{s}}, \\
\text { potential: } & t=\left(M v^{2}\right)^{-1} T_{\mathrm{u}}, \vec{x}=(M v)^{-1} \vec{X}_{\mathrm{s}}, \\
\text { ultrasoft: } & t=\left(M v^{2}\right)^{-1} T_{\mathrm{u}}, \vec{x}=\left(M v^{2}\right)^{-1} \vec{X}_{\mathrm{u}} .
\end{aligned}
$$


The scaling of the fields is derived by normalising the propagator terms to be of order $v^{0}$. In order to maintain velocity power counting, corrections of order $v$ or higher in the thus rescaled Lagrangean must be treated as insertions. Particles in the various régimes couple: On-shell (potential) quarks radiate bremsstrahlung (ultrasoft) gluons. In general, one must allow all couplings between the various régimes which obey "scale conservation" for energies and momenta. This excludes for example the coupling of two potential quarks $\left(T \sim M v^{2}\right)$ to one soft gluon $\left(q_{0} \sim M v\right)$, but not to two soft gluons.

Amongst the fields introduced, two (six) interactions are allowed for example within and between the various régimes for the scalar coupling in the Coulomb (Lorentz) gauge whose $v$ counting is read up from the rescaled Lagrangean [9, 10, 11, see table 1. Albeit both describing interactions with physical gluons, soft and ultrasoft couplings occur at different orders in $v$, and obey different multipole expansion rules. Double counting is prevented because in addition to most of the propagators, all vertices involve different multipole expansions. Because the vertex rules for the soft régime count powers of $v$ with respect to the soft régime, one retrieves there the velocity power counting of Heavy Quark Effective Theory [12, 13]. HQET becomes a sub-set of NRQCD, complemented by interactions between soft (HQET) and potential or ultrasoft particles. In NRQCD with

Table 1: Velocity power counting and vertices for the interaction Lagrangean $-g Q^{\dagger} A_{0} Q$ in the Lorentz gauge. In the Coulomb gauge, only the first two diagrams exist. Zigzagged, dashed and wiggly lines represent $A_{\mathrm{s}}^{\mu}, A_{\mathrm{p}}^{\mu}$ and $A_{\mathrm{u}}^{\mu}$, double and single lines $Q_{\mathrm{s}}$ and $Q_{\mathrm{p}}$, respectively.

\begin{tabular}{|c|c|c|c|c|c|c|}
\hline Vertex & $\begin{array}{l}A_{0} \\
A_{0}\end{array}$ & $\begin{array}{l}A_{0} \\
I_{1}\end{array}$ & \{ & $\$$ & 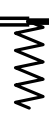 & $\xi$ \\
\hline$v$ power & $\frac{1}{\sqrt{v}}$ & $\sqrt{v}$ & $v^{0}$ & $v^{0}$ & $v^{0}$ & $v$ \\
\hline
\end{tabular}

two potential quarks as initial and final states, the power counting in a soft sub-graph has to be transfered to the potential régime [9, 10]. Because soft loop momenta scale like $\left[\mathrm{d}^{4} k_{\mathrm{s}}\right] \sim v^{4}$, while potential ones like $\left[\mathrm{d}^{4} k_{\mathrm{p}}\right] \sim v^{5}$, each largest sub-graph which contains soft particles is enhanced by an additional factor $\frac{1}{v}$.

Matching between NRQCD and QCD proved that the soft quark and gluon are indispensable to reproduce the correct structure of collinear (infrared) divergences of the two gluon exchange contribution to Coulomb scattering between non-relativistic particles near threshold in a toy model and confirmed the proposed counting rules [9].

Because NRQCD is a well-defined field theory of quarks and gluons, one can investigate its renormalisation group equations under the assumption that perturbation theory is applicable at all scales. NRQCD must reproduce the QCD $\beta$ function with $N_{\mathrm{F}}$ light quarks below the scale $M$. The result thus already anticipated,

$$
\beta_{\mathrm{NRQCD}}=-\frac{g_{\mathrm{R}}^{3}}{(4 \pi)^{2}}\left[\frac{11}{3} N-\frac{2}{3} N_{\mathrm{F}}\right]
$$


to lowest order for the gauge group $\mathrm{SU}(N)$, the prime goal of this exercise [10] is not the result but to provide further insight: The Lorentz gauge is a legitimate gauge choice and simplifies renormalisation. The renormalised coupling strengths of all interactions steming from expanding the same term in the Lagrangean in the various régimes are the same, except that they have to be taken at different scales. Although the number of vertices is increased, the number of independent couplings is not. The soft régime is indispensable for NRQCD to describe especially the correct running coupling for the Coulomb gluons, as their sole contribution in the vacuum polarisation comes from the propagation of soft (on-shell) gluons in the loop. The computation also confirms the validity of the power counting proposed and is slightly simpler than its QCD counterparts. The non-Abelian nature of the gauge field enters to lowest order only via the non-zero contribution of the three gluon vertex to the vacuum polarisation. Non-Abelian vertex contributions are down by at least one power in $v$ compared to Abelian vertex corrections and bare vertices. The quark self energy and vertex renormalisations differ from its QCD counterpart, but the Slavnov-Taylor identities hold.

From a technical point of view, the number of possible diagrams in power counted NRQCD is considerably larger than in the original theory because there are at least six vertices per interaction allowed by scale conservation, cf. table 1 . The calculation is greatly simplified by diagrammatic rules [10] which follow from the homogeneity property of dimensional regularisation. That integrals without scales set by external momenta or energies in the denominator vanish in dimensional regularisation,

$$
\int \frac{\mathrm{d}^{d} q}{(2 \pi)^{d}} q^{\alpha}=0
$$

allows one to recognise the majority of NRQCD (and threshold expansion [1]) graphs as zero to all orders in $v$ just by drawing them, independently of details of the vertices involved. The concept is sensitive only to the multipole expansion of the vertices and independent of insertions and the chosen gauge. Only the denominators of the graph lowest order in $v$ have to be looked at. It is usually only necessary to consider a sub-set of the whole diagram. One assigns the loop and external momenta to it, taking into account multipole expansions, and writes out the denominators (i.e. inverse propagators). If by shifting integration variables, one arrives at an energy or momentum integral without scale, the diagram vanishes, as do any diagrams which contain it as a sub-graph. As an example, the following rule may be quoted, which states that a potential gluon coupled to two soft particles, quark or gluon, vanishes because there is no scale in the $q_{0}$ part of the loop integration, even when potential or ultrasoft particles couple to the Coulomb gluon:

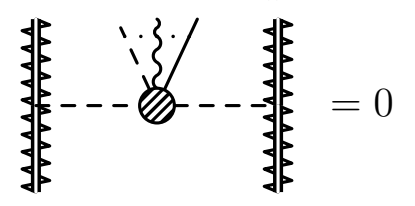

In the case of the NRQCD $\beta$ function, the number of vertices for each of the couplings of table 1 is reduced from three or more to one, and the number of vacuum polarisations also 
from three to one. This result holds for any gauge and any interaction involving loops with three particles, showing the general usefulness of these rules.

For a derailed account of the points outlined in this contribution, I refer to [9, 10] and references therein. In conclusion, a complete velocity power counting of NRQCD has been suggested. The results are readily generalised to any effective field theory with two or more infrared scales.

\section{References}

[1] W. E. Caswell and G. P. Lepage: Phys. Lett. B 167, 437 (1986).

[2] G. T. Bodwin, E. Braaten and G. P. Lepage: Phys. Rev. D 51, 1125 (1995); Phys. Rev. D 55, 5853 (1997).

[3] G. P. Lepage et al.: Phys. Rev. D 46, 4052 (1992).

[4] M. Beneke and V. A. Smirnov: Asymptotic Expansion of Feynman Integrals near Threshold; CERN-TH/7-315, hep-ph/9711391, 1997.

[5] M. Luke and A. V. Manohar: Phys. Rev. D 55, 4129 (1997).

[6] B. Grinstein and I. Z. Rothstein: Phys. Rev. D 57, 78 (1998).

[7] M. Luke and M. J. Savage: Phys. Rev. D 57, 413 (1998).

[8] P. Labelle: Effective Field Theories for QED Bound States: Extending Nonrelativistic QED to Study Retardation Effects; MCGILL-96-33, hepph/9608491, 1996 .

[9] H. W. Grießhammer, Threshold Expansion and Dimensionally Regularised NRQCD; NT@UW-98-3, hep-ph/9712467, 1997 (to be published in Phys. Rev. D 58).

[10] H. W. Grießhammer, Power Counting and $\beta$ Function in NRQCD; NT@UW98-22, hep-ph/9810235, 1998 .

[11] H. W. Grießhammer, The Soft Régime in NRQCD; NT@UW-98-12, hepph/9804251, 1998 (Proceedings of the Workshop "Nuclear Physics With Effective Field Theories" at Caltech, 26th - 27th February 1998, eds. R. Seki, U. van Kolck and M. J. Savage, World Scientific).

[12] N. Isgur and M. B. Wise: Phys. Lett. B 232, 113 (1989).

[13] N. Isgur and M. B. Wise: Phys. Lett. B 237, 527 (1990). 